\title{
Analysis of Grain Boundary Dynamics Using Event Detection and Cumulative Averaging
}

\author{
A. Gautam, C. Ophus, F. Lançon ${ }^{1}$ and U. Dahmen \\ National Center for Electron Microscopy, LBNL, Berkeley, CA 94720 USA \\ ${ }^{1}$ Laboratoire de Simulation Atomistique (L_Sim), SP2M, INAC, CEA, 38054 Grenoble, \\ France
}

\begin{abstract}
To analyze extended time series of high resolution images, we have employed automated frame-by-frame comparisons that are able to detect dynamic changes in the structure of a grain boundary in Au. Using cumulative averaging of images between events allowed high resolution measurements of the atomic relaxation in the interface with sufficient accuracy for comparison with atomistic models. Cumulative averaging was also used to observe the structural rearrangement of atomic columns at a moving step in the grain boundary. The technique of analyzing changing features in high resolution images by averaging between incidents can be used to deconvolute stochastic events that occur at random intervals and on time scales well beyond that accessible to single-shot imaging.
\end{abstract}

Keywords: HRTEM; HAADF; dynamics; atomic structure; grain boundary; interface; simulation; step; time average; aberration correction; electron detector

\section{Introduction}

The motion of interfaces in solids is often controlled by the nucleation and propagation of steps [1-3]. However, nucleation events are stochastic in nature and thus difficult to follow dynamically by in-situ observation. For example the migration of grain boundaries during capillary shrinkage of island grains in Au typically follows non-parabolic kinetics and proceeds erratically, so that dormant periods alternate with rapid bursts of activity or structural changes [4].

Observations of interface dynamics are usually made by diffraction contrast or high resolution imaging at video rates [5-9]. This limits the spatial and temporal resolution of the observations to features that are large enough to be resolved and slow enough to be recorded. These constraints are particularly restrictive for stochastic events such as step motion or fluctuation between different metastable states. By their nature, such events occur at random intervals and thus do not lend themselves to time-resolved pump-probe experiments or stroboscopic techniques $[10,11]$. The fast rate of such transitions usually makes it impossible to record them directly, and thus high resolution images often represent a superposition of different structural states, resulting in image blurring of the critical features, even in atomic resolution images with high $\mathrm{S} / \mathrm{N}$ ratio. 
However, thanks to recent advances in electron microscopy, it has become possible to record high resolution images with improved $\mathrm{S} / \mathrm{N}$ ratio and at higher rates than previously feasible. Here we show how it is possible to deconvolute stochastic events by applying statistical averaging techniques to time-resolved image sequences. This allows us to analyze the evolution of atomic structure with a precision that is sufficient for comparison with atomistic simulations.

Statistical techniques of image processing are commonly used to enhance the $\mathrm{S} / \mathrm{N}$ in high-resolution imaging, typically by averaging over periodically spaced features in the spatial domain, such as structural units in a grain boundary [e.g. 12], unit cells in a beam sensitive crystal [e.g. 13], or randomly-distributed features such as proteins for singleparticle analysis in cryo-electron microscopy [14]. In the temporal domain, statistical averaging is most commonly used in pump-probe experiments, which allow cumulative data acquisition from many low-dose images or diffraction patterns $[11,15]$.

In the present study, we investigate the atomic structure of the $(001) /(110)$ grain boundary in $\left.90^{\circ}<110\right\rangle$ bicrystals of $\mathrm{Au}$. This boundary is incommensurate because the repeat periods of lattice planes of the two grains that are aligned across the boundary are in the ratio of $1: \sqrt{2}$. For gold bicrystals, an analysis based on the Frenkel-Kontorova model found atomic relaxations characteristic of interfaces capable of superglide behavior [16-18].

Because of a strong anisotropy in the energy of this grain boundary, its migration is controlled by the nucleation and propagation of steps [3]. In order to understand this boundary in more detail, we have analyzed its dynamic behavior by following the motion of defects during observation by atomic resolution electron microscopy at room temperature. Thus, even though $300 \mathrm{kV}$ electrons are well below the knock-on threshold for $\mathrm{Au}$ [19], the dynamic effects observed here are stimulated by the electron beam [6]. As both grains in the bicrystal have a $\langle 110\rangle$ surface, surface energy does not provide a driving force for boundary motion, and as a result, the observed features are most likely fluctuations around equilibrium. However, such structural fluctuations are expected to be important for thermally activated processes during grain coarsening, step motion and deformation.

\section{Experimental Procedure}

The samples were prepared by physical vapor deposition of high purity Au onto heated single crystal Ge substrates [20]. Due to preferred heteroepitaxial alignment, thin films grew as two symmetry-equivalent $\langle 110\rangle$ variants, rotated $90^{\circ}$ relative to each other in the mazed bicrystal structure, with grain boundaries of fixed misorientation but variable inclination. The Ge substrate was later dissolved in a 1:1 solution of hydrofluoric acid and hydrogen peroxide, resulting in freestanding Au films of about $4 \mathrm{~nm}$ thickness.

Thin film samples were imaged by high resolution electron microscopy in the TEAM microscope [15], using a direct electron detector [21,22], and analyzed with custom routines written in the MATLAB programming language. Dynamic structural changes were detected by applying an edge detection filter in the time domain, using the procedure described in the Appendix. 
High resolution images were recorded in the aberration-corrected TEAM microscope at $300 \mathrm{kV}$, using both, HRTEM and HAADF imaging. HRTEM images were recorded with the TEAM detector, a direct electron detector developed to optimize the efficiency and dynamic range of electron detection in aberration-corrected electron microscopes [21,22]. The detector was used at 90 frames/s and 1024 x 512 pixels per frame. The microscope was operated at $300 \mathrm{kV}$ with $\mathrm{Cs}=-10 \mu \mathrm{m}$ and $\mathrm{Cc}<20 \mu \mathrm{m}$ (typically $\sim 5 \mu \mathrm{m}$ ). In HAADF, rapid scans were obtained at a resolution of 32 pixels/nm and a dwell time of $0.8 \mu \mathrm{s} /$ pixel.

\section{Results and Discussion}

Figure 1 demonstrates the approach of event detection and cumulative averaging by application to an extensive series of HRTEM images of the $(001) /(110)$ grain boundary in Au. A sequence of about 6,000 images was recorded at a rate of $90 \mathrm{fps}$ using a direct electron detector [23]. Each frame was 512 x 1024 pixels in size. A typical image from this series is shown in Figure 1a. The $\mathrm{S} / \mathrm{N}$ ratio is poor but sufficient to apply an algorithm that compares sequential images to detect any changes in structure as well as image shifts due to drift or vibration. After correcting for image shifts, randomly spaced structural changes can be found automatically and plotted as a function of time. The graph in Figure 1e shows events plotted in a series of spikes. Taller spikes represent larger changes (see appendix). Between spikes, the structure remains unchanged. By cumulative averaging of all images between events, we obtain high-quality HRTEM micrographs of the same quality as images obtained by conventional single exposures see Figure 1b. Such images can be color-coded by their local coordination to indicate relaxations in the interface, as shown in Figure 1c. The rectangular region outlined in white encompasses the nearly vertical (100)/(011) segment of the boundary. This region is shown magnified in Figure 1d, which highlights a structural change using an RGB overlay of two images obtained by cumulative averaging between events. The atomic rearrangements are seen in red. The shift of a single close-packed plane at the top right is part of the chevron reconstruction, which has been found to occur in low-stacking fault materials at the intersection of a grain boundary with a free surface [24]. The rearrangement in the center of the image is a structural change that is characteristic for the high-energy positions in the atomic structure of this boundary [25]. 

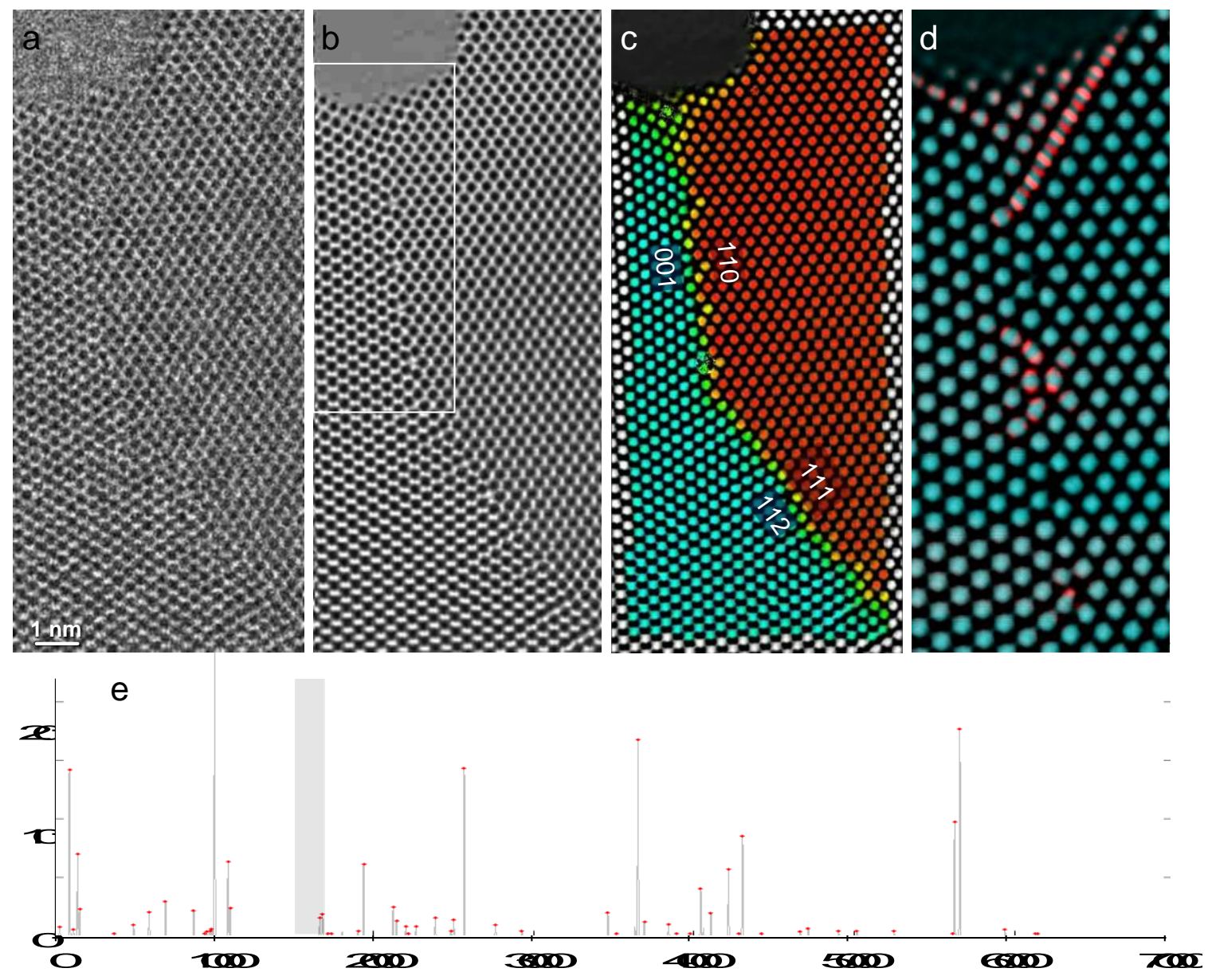

Figure 1 -Application of event detection and cumulative averaging to an HRTEM image series of 6,000 frames recorded with a direct electron detector at a rate of $90 \mathrm{fps}$. An individual $512 \times 1024$ image from this series is shown in a). Cumulative averaging between events yields high quality images $(b)$, which can be used to analyze the nature of atomic motion by highlighting changes in local coordination (c) and local motion of atomic columns $(d)$ in a magnified view of the $(001) /(110)$ boundary segment framed in (b). Automated comparison between sequential images detects structural events as spikes in the correlation coefficient plotted over the entire sequence (e). The gray bar illustrates that a 2 s exposure would tend to straddle one or several events, thus convoluting different states in a single image.

Note that the $(001) /(110)$ facet in this image is bounded at the top by the edge of the sample and at the bottom by a (111)/(11-2) facet. During observation, the chevron reconstruction at the top edge is very active. Structural changes in this region such as those seen in Figure 1c are responsible for most of the events plotted in the graph in Figure 1e. By comparison, the (111)/(11-2) facet is completely stable and does not undergo any changes during the entire period of observation. The structure of this facet has been analyzed in terms of a local dissociation into a 9R structure involving Shockley partial dislocations on one side of the interface [26]. These defects are visible in Figure $1 \mathrm{~b}$ as an apparent bending of close-packed planes in the grain to the left of the lower, inclined facet. 
The localized changes in the center of the image in Figure 1d are characteristic of the intrinsic structure of the $(001) /(110)$ boundary and are commonly observed during high resolution imaging [25]. A magnified view of this segment of the boundary is shown in Figure 2, which highlights relevant features in color. The image in a) shows a frame from an event-averaged movie that color-codes local changes in coordination. Atomic columns that deviate from their coordination in either crystal are seen in yellow and green. The image in b) shows the same area, highlighting local atomic motion between events in red. The image in c) shows part of an atomistic simulation of this structure using a rational approximant [25] to calculate the energy of atomic sites. Sites of relatively high energy are indicated in red. Notice that the atomic motion in b) is confined to the high-energy sites in the center between the two five-sided units that are outlined with dashed circles. These sites remained stationary throughout this experiment, but are often found to undergo characteristic fluctuations during dynamic observation of longer segments of this interface.
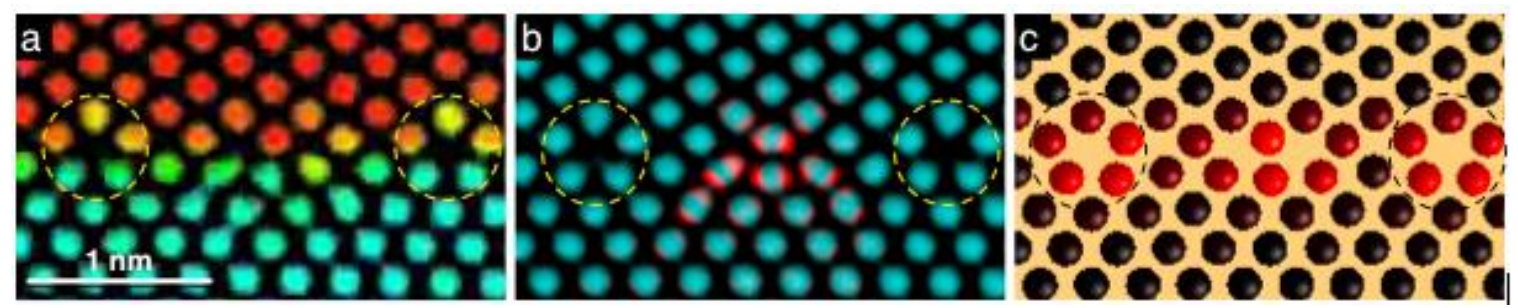

Figure 2 - Magnified view of central area in Figure 1d showing structural rearrangements in (001)/(110) facet. The image in a) is from an event-averaged movie showing local coordination in color. Red and cyan correspond to the two grain orientations; green and yellow indicate local distortions in the boundary. The same area is shown in b) with dynamic atomic column shifts highlighted in red. In c) the relative energy of atomic sites in an atomistic model shows high-energy sites in red. Dashed circles outline characteristic high-energy sites that remain stable in this image sequence.

In order to measure the atomic structure of this boundary in more detail, we have used the hull function, which provides a statistical representation of the atomic column shifts in each grain due to the periodic influence of the opposite grain [17,27,28]. Such measurements can be made cumulatively by adding data from many images of many boundaries [25]. Although structural changes in the interface, such as those highlighted in the center of Figure 1c, are due to the influence of the electron beam, the atomic structures between events will all be part of the states that constitute the hull function. Thus, atomic position measurements made on either side of an event contribute to the hull plot, while measurements from images that straddle an event must be avoided.

To obtain a sufficiently fine sampling of atomic relaxations in this boundary, it is necessary to measure a longer segment of the interface than that shown in Figures 1 and 2. An analysis of such a boundary is illustrated in Figure 3. The HRTEM image shows the first in a time sequence of 150 micrographs recorded at intervals of 0.5 seconds. The displacements of atomic columns relative to their unrelaxed position in the perfect lattice are shown for the two atomic planes immediately adjacent to the interface. Arrows indicate the direction and magnitude of the displacements for the (110) plane in the upper grain and the (001) plane in the lower grain. A cumulative plot of these displacements is 
presented in the graphs at the right, mapping relaxations in the atomic plane immediately adjacent to the boundary, for the upper grain (red) and the lower grain (blue), in the direction parallel to the boundary (left) and perpendicular to the boundary (right).
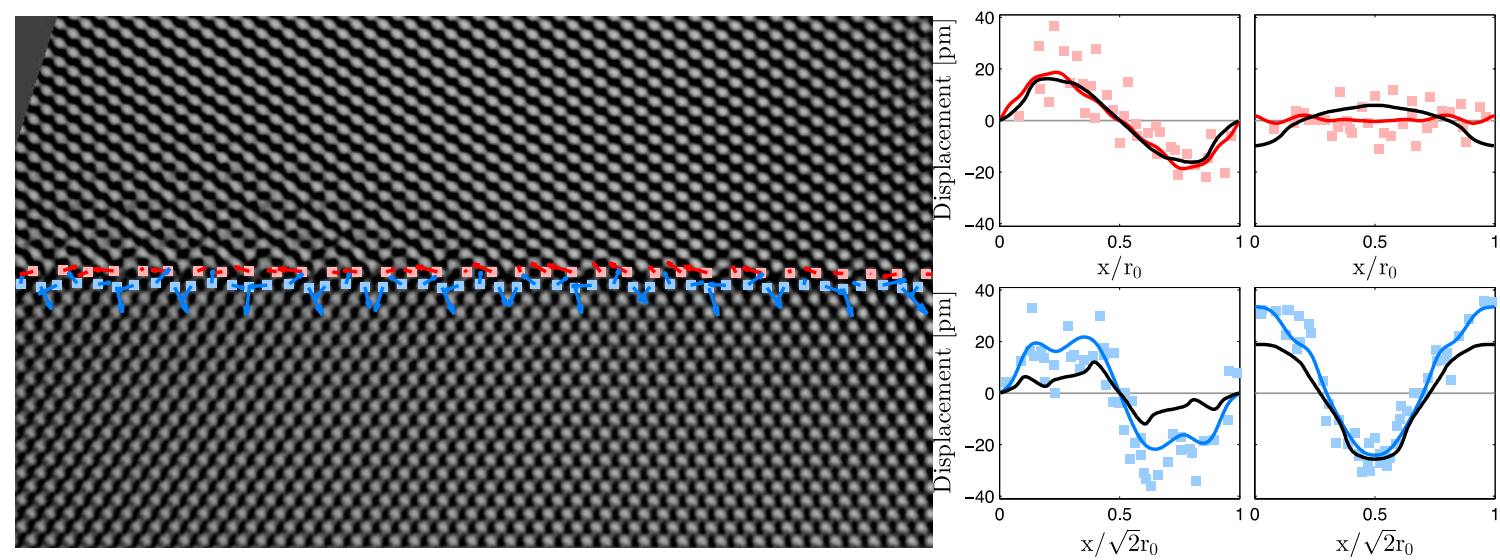

Figure 3 - Measurement of hull plot from 150 sequential images of the (001)/(110) boundary. In the HRTEM image, atomic column displacements in the planes adjacent to the boundary are shown as red and blue arrows for the upper and lower grain (scaled x10). Displacement components parallel and perpendicular to the boundary are shown in the left and right columns, respectively. Least squares data fits shown as colored lines and model structure shown as black lines.

The pattern that emerges characterizes the atomic relaxation at the boundary by its deviation from a straight line at $\mathrm{y}=0$ (the unrelaxed configuration). This pattern (shown in color) can be compared with that predicted from atomistic simulations [17] (shown in black). In the present case, this comparison agrees in the general trend but shows some discrepancies that could be due to the use of an interatomic potential to model the potential energy of the grain boundary, or an experimental artifact manifested in a moiré pattern of growing intensity at the left side of the upper grain as the experiment progresses. Since the origin of this artifact has yet to be resolved, we use these measurements mainly to illustrate the potential of this approach for quantitative analysis by cumulative measurements on many images [25].

The technique of event detection and cumulative averaging has also been applied to HAADF imaging. Figure 4 shows a series of rapid scans obtained at a rate of $2 \mathrm{fps}$, resulting in a sequence of 100 images of poor quality but with sufficient $\mathrm{S} / \mathrm{N}$ to align sequential images to within one pixel of each other to correct for drift or vibration. 


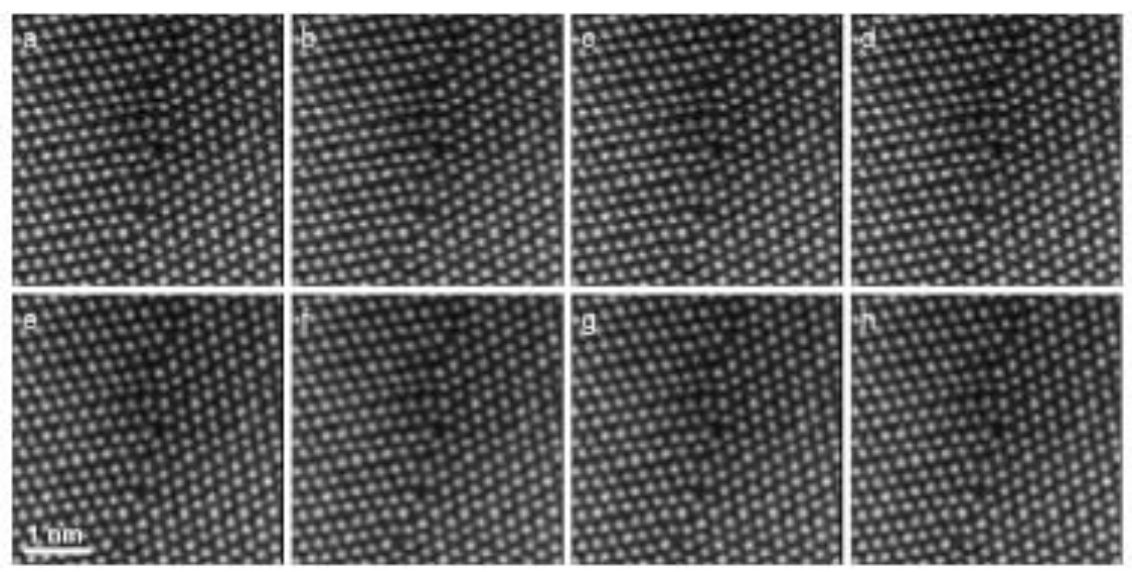

Figure 4 - HAADF STEM images of a step in a grain boundary. The images are representative frames from a sequence of 100 rapid scans of the same area. Although the individual image quality is poor, frame-by-frame comparison uncovered a structural event somewhere in the middle of the sequence. Cumulative averaging before and after the event results in the images shown in Figure 5.

By averaging over image sequences during stable periods, we obtained the micrographs in Figure 5, which reveal the structure of a step in the $(001) /(110)$ interface. The micrographs in a) and b) show cumulative averages of the step structure before and after motion. The image in c) is an RGB overlay of these two images, highlighting the structural change during step motion as a shift in atomic columns from cyan to red.

Atomic coordinates $x$ and $y$ extracted from the experimental image in a) were used to construct a structural model. Atomic interactions were modeled by an embedded-atom method (EAM) potential [29]. A layer of ten atomic planes was obtained by minimizing the total potential energy with free surface conditions along $\mathrm{z}$, and with fixed boundary conditions for $\mathrm{x}$ and $\mathrm{y}$ coordinates of the atoms located at the image edges. The resulting model in d) shows the atomic arrangement at the step, with color-coding indicating the $\mathrm{z}$ component of the atomic columns. The ABAB stacking of fcc crystals along [1-10] (the direction of the projection) is indicated by the yellow/orange/red contrast, denoting a relative shift of $1 / 4[1-10]$ between yellow and orange or between red and orange. Yellow and red are separated by the period $1 / 2[1-10]$ as in a perfect crystal.

At the step, three (001) planes of one grain meet four (110) planes of the other grain. To avoid a high-energy stacking fault due to this $3 \mid 4$ mismatch, a screw dislocation is required along the direction of projection. So starting at a yellow atom in Fig. $5 \mathrm{~d}$, a helical path cycling twice around the screw dislocation can be drawn through orange atoms, ending at a red atom which is equivalent by periodicity to the initial yellow atom. The screw dislocation ensures that the 1/4[1-10] shift required by the AB stacking sequence is maintained at the boundary on both sides of the step. A detailed analysis of the energy and path of the atomic motion lies outside the scope of the present manuscript, and will be presented elsewhere. 

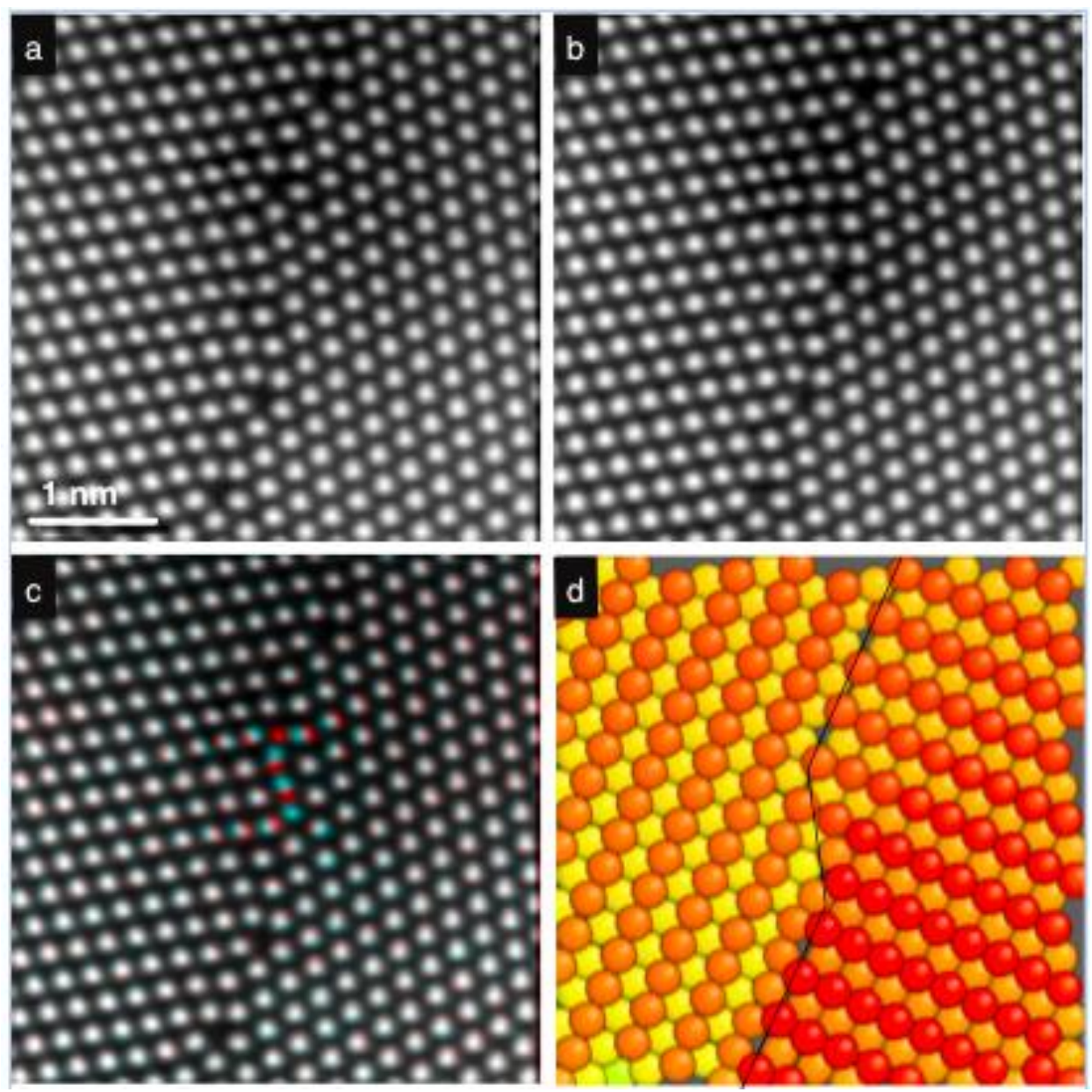

Figure 5 - Structure of a moving step. Atomic resolution HAADF STEM images show the observed structure before and after motion (a) and (b). The RGB overlay of the two images in (c) highlights the atomic column displacements involved in the step motion. The model in (d) shows the structure of the step, representing the AB stacking of the crystal along the beam direction in color. The difference between red/orange and orangelyellow is the $1 / 4<110\rangle$ change in stacking between A and B columns.

Although the motion of steps plays an important role in the migration of faceted interfaces [3] a clear understanding of the structure, energetics and mobility of steps is still lacking. The first HRTEM observations of moving boundaries in $\mathrm{Au} \mathrm{[6]} \mathrm{revealed}$ microfaceting in a 110 twist boundary and step motion in an incommensurate boundary near (120)/(311) in Au. However, the step structure was not resolved, and because some atoms undergo relatively large shifts, it was concluded that the motion involves a highly disordered state. Subsequent observations of step motion in a symmetrical boundary in $\mathrm{Al}$ at 523K [9] showed fluctuations of a step associated with a dislocation. Similar observations of tilt grain boundaries in Au at $823 \mathrm{~K}$ offered evidence of collective motion involving up to several hundred atoms [7]. Grain boundary migration driven by surface energy was found to proceed erratically, while grain boundaries under capillary forces exhibited rapid reversible motions [8]. Images of boundaries that remained stable for extended periods were used for temporal averaging from a series of video frames. The extensive set of experimental observations presented in [8] led to the conclusion that 
grain boundaries could move by three major mechanisms - glide, ledge motion and cooperative shuffles and that these modes were related to the crystallographic character of the boundary. However, the accuracy of the observations was not yet sufficient for quantitative comparisons with atomistic simulations.

The approach described here offers a tool for critical comparisons between observed and simulated grain boundary structures, and thus could contribute to a better understanding of the relationship between atomic structure and properties of grain boundaries. Together with temporal statistics on events such as fluctuation between metastable structures, this offers great potential for further advances in our understanding of the mechanisms and dynamics of defects in interfaces.

\section{Summary and conclusions}

We have shown how event detection and cumulative image averaging can help with a detailed analysis of high resolution image series of stochastic dynamic events. The approach is illustrated for HRTEM and HAADF images of structural changes that take place in a $90^{\circ}<110>$ tilt grain boundary in Au under the influence of the electron beam. By splitting a series of images at sudden events such as step motion or fluctuations around high-energy structural states of the interface, image blurring due to overlapping structures can be avoided. This makes it possible to analyze the atomic structure of dynamic defects with sufficient accuracy to facilitate quantitative comparison with atomistic simulations.

\section{Acknowledgement}

This work is supported by the Director, Office of Science, Office of Basic Energy Sciences, of the U.S. Department of Energy under Contract No. DE-ACO3-76SFOOO98, and by the Direction des Sciences de la Matière of the CEA.

\section{Appendix}

Thin film samples were imaged by high resolution electron microscopy in the TEAM microscope [15], using a direct electron detector [21,22], and analyzed with custom routines written in the MATLAB programming language. Since the frame rate was extremely high, we have developed the following reconstruction/smoothing routine. Intensity spikes or steps for every pixel were detected by convolution filtering along the time dimension. First, a numerical filter (kernel) was defined, and then the absolute convolution between this kernel and the measured experimental data was performed. An efficient kernel for detecting steps is given by the first derivative of a Gaussian distribution, which varies as $t \exp \left(t^{2} / 2^{2}\right)$, where $t$ is the time in frames, and $\tau$ is the bandwidth of the filter kernel in frames [30]. Events were detected by convolving this function with the measured intensity along the time dimension, taking the absolute magnitude, and then determining the local maxima with magnitudes above a threshold value (determined by inspection). The final reconstructed signal is defined as the median values of all measurements performed between each adjacent pair of steps detected. Median statistics were used because of their superior robustness against outliers. 
Figure 6 shows this procedure schematically at a single pixel using synthetic data with random Poisson noise applied. Two cases are presented, with the left figures having an average of 100 counts per frame, and the right figures having an average of 10 counts per frame. The top graphs show the ideal underlying signal (black), and a noisy measurement of it (gray). The center frames show the absolute magnitude of the step detection filter (convolution kernel inset into figure) and its detected peak values. The bottom frames show a comparison between the original noise-free data (black) and the reconstructed signal (blue). As expected, the left side example with a signal to noise of approximately 10 is reconstructed nearly perfectly, with only a single minor step missed. By contrast, reconstructing the example to the right (with a signal-to-noise of approximately 3.1) requires a filter with a much larger bandwidth. Several steps have been missed, and the step boundaries show some errors. In particular, the final spike in the data is narrower than the filter bandwidth and one side of it is completely missed. However, the reconstruction is still quite accurate overall.
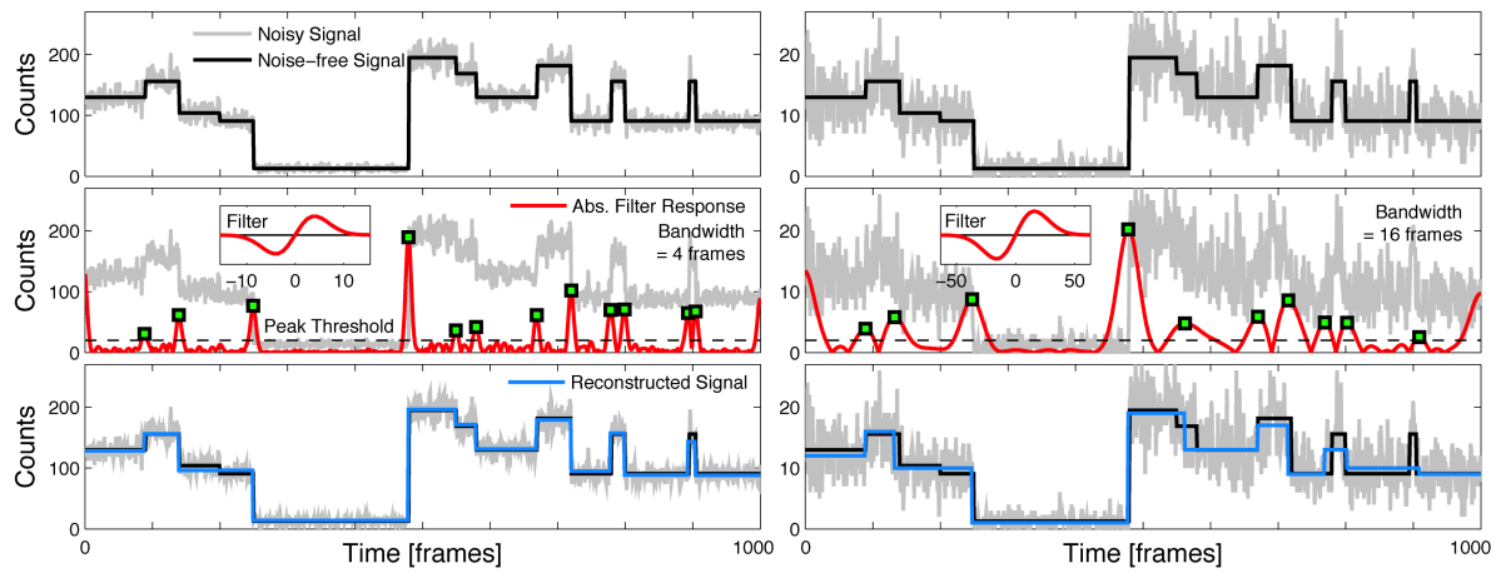

Figure 6: Illustration of automated procedure used for event detection comparing the accuracy of the technique for high $S / N$ (left column) and low $S / N$ (right column). The filter kernel is shown in the central row. The accuracy of the technique is seen in the bottom row by comparing the actual noise-free data (black line) with the reconstructed signal (blue line). The noisy data are shown in gray.

In the experimental datasets, we have additional information given by neighboring pixels. All detected steps occurring at neighboring pixels and within a time range $\tau$, were forced to the same timing for a common event. This improves the reconstruction quality significantly, even for very noisy input data. Thus a good estimate of the local intensity was recovered even for these very noisy signals without sacrificing temporal resolution, as long as the event density remained low (time between events must be somewhat higher than the detection filter bandwidth).

\section{References}

[1] H. Gleiter, The mechanism of grain boundary migration, Acta Metall. 17 (1969).

[2] A.P. Sutton, R.W. Balluffi, Interfaces in Crystalline Materials, Oxford University 
Press on Demand, 1995.

[3] C.P. Race, J. Von Pezold, J. Neugebauer, Role of the mesoscale in migration kinetics of flat grain boundaries, Phys Rev B. 89 (2014) 214110.

doi:10.1103/PhysRevB.89.214110.

[4] T. Radetic, C. Ophus, D.L. Olmsted, M. Asta, U. Dahmen, Mechanism and dynamics of shrinking island grains in mazed bicrystal thin films of Au, Acta Materialia. 60 (2012) 7051-7063. doi:10.1016/j.actamat.2012.09.012.

[5] S.E. Babcock, R.W. Balluffi, Grain boundary kinetics-I. In situ observations of coupled grain boundary dislocation motion, crystal translation and boundary displacement, Acta Metall. 37 (1989) 2357-2365. doi:10.1016/00016160(89)90033-3.

[6] K.L. Merkle, L.J. Thompson, Atomic-scale observation of grain boundary motion, Mater Lett. 48 (2001) 188-193. doi:10.1016/S0167-577X(00)00301-3.

[7] K. Merkle, L. Thompson, F. Phillipp, Collective effects in grain boundary migration, Phys Rev Lett. 88 (2002) 225501.

doi:10.1103/PhysRevLett.88.225501.

[8] K. Merkle, L. Thompson, F. Phillipp, In-situ HREM studies of grain boundary migration, Interface Sci. 12 (2004) 277-292.

[9] K. Merkle, L. Thompson, F. Phillipp, Thermally activated step motion observed by high-resolution electron microscopy at a (113) symmetric tilt grain-boundary in aluminium, Phil Mag Lett. 82 (2002) 589-597.

doi:10.1080/0950083021000038074.

[10] W. King, G.H. Campbell, A. Frank, B. Reed, J. Schmerge, B. Siwick, et al., Ultrafast electron microscopy in materials science, biology, and chemistry, J Appl Phys. 97 (2005) -. doi:10.1063/1.1927699.

[11] A.H. Zewail, 4D ultrafast electron diffraction, crystallography, and microscopy, Annu. Rev. Phys. Chem. 57 (2006) 65-103. doi:10.1146/annurev.physchem.57.032905.104748.

[12] K. Merkle, D. Smith, Atomic Structure of Symmetric Tilt Grain Boundaries in NiO, Phys Rev Lett. 59 (1987) 2887-2890. doi:10.1103/PhysRevLett.59.2887.

[13] M.C. Sarahan, Q.M. Ramasse, N.D. Browning, Data Processing Techniques for Aberration-Corrected STEM, Microsc Microanal. 16 (2010) 112-113. doi:10.1017/S1431927610055327.

[14] J. Frank, Three-Dimensional Electron Microscopy of Macromolecular Assemblies : Visualization of Biological Molecules in Their Native State, Oxford University Press, 2006.

[15] U. Dahmen, R. Erni, V.R. Radmilovic, C.F. Kisielowski, M.-D. Rossell, P. Denes, Background, status and future of the Transmission Electron Aberrationcorrected Microscope project, Philos T R Soc A. 367 (2009) 3795-3808. doi:10.1098/rsta.2009.0094.

[16] F. Lancon, J. Ye, D. Caliste, T. Radetic, A.M. Minor, U. Dahmen, Superglide at an Internal Incommensurate Boundary, Nano Lett. 10 (2010) 695-700. doi:10.1021/n1903885p.

[17] F. Lancon, Aubry transition in a real material: Prediction for its existence in an incommensurate gold/gold interface, Europhys Lett. 57 (2002) 74-79.

[18] F. Lancon, J.M. Penisson, U. Dahmen, Quasicrystalline gold interface with a 
hypo-friction property, Europhys Lett. 49 (2000) 603-609.

[19] R.F. Egerton, P. Li, M. Malac, Radiation damage in the TEM and SEM, Micron. 35 (2004) 399-409. doi:10.1016/j.micron.2004.02.003.

[20] K.H. Westmacott, S. Hinderberger, U. Dahmen, Physical vapour deposition growth and transmission electron microscopy characterization of epitaxial thin metal films on single-crystal Si and Ge substrates, Philos Mag A. 81 (2001) 1547-1578.

[21] P. Denes, J.-M. Bussat, Z. Lee, V.R. Radmilovic, Active Pixel Sensors for electron microscopy, Nucl Instrum Meth A. 579 (2007) 891-894. doi:10.1016/j.nima.2007.05.308.

[22] M. Battaglia, D. Contarato, P. Denes, D. Doering, T. Duden, B. Krieger, et al., Characterisation of a CMOS active pixel sensor for use in the TEAM microscope, Nuclear Inst. and Methods in Physics Research, A. 622 (2010) 669677. doi:10.1016/j.nima.2010.07.066.

[23] M. Battaglia, D. Contarato, P. Denes, D. Doering, P. Giubilato, T.S. Kim, et al., A rad-hard CMOS active pixel sensor for electron microscopy, Nucl Instrum Meth A. 598 (2009) 642-649. doi:10.1016/j.nima.2008.09.029.

[24] T. Radetic, F. Lancon, U. Dahmen, Chevron defect at the intersection of grain boundaries with free surfaces in Au, Phys Rev Lett. 89 (2002) 085502. doi:10.1103/PhysRevLett.89.085502.

[25] A. Gautam, C. Ophus, F. Lancon, V.R. Radmilovic, U. Dahmen, Atomic structure characterization of an incommensurate grain boundary, Acta Materialia. 61 (2013) 5078-5086. doi:10.1016/j.actamat.2013.04.028.

[26] D. Medlin, S.M. Foiles, D. Cohen, A dislocation-based description of grain boundary dissociation: Application to a 90 degrees tilt boundary in gold, Acta Materialia. 49 (2001) 3689-3697.

[27] T. Kawaguchi, H. Matsukawa, Dynamical frictional phenomena in an incommensurate two-chain model, Phys Rev B. 56 (1997) 13932-13942.

[28] S. Aubry, P. Ledaeron, The Discrete Frenkel-Kontorova Model and its Extensions I. Exact Results for the Ground-States, Physica D. 8 (1983) 381-422.

[29] T. Deutsch, P. Bayle, F. Lancon, J. Thibault, Computer simulation of $\mathrm{Au}(001) / \mathrm{Ni}$ multilayers: comparison with experiments, J Phys-Condens Mat. 7 (1999) 64076421. doi:10.1088/0953-8984/7/32/007.

[30] J. Canny, A computational approach to edge detection, Pattern Analysis and Machine Intelligence, IEEE Transactions on. (1986) 679-698. 